\title{
C-reactive protein: a critical update
}

\author{
Mark B. Pepys and Gideon M. Hirschfield \\ Centre for Amyloidosis and Acute Phase Proteins, Department of Medicine, Royal Free and University College Medical School, \\ London, United Kingdom
}

J. Clin. Invest. 111:1805-1812 (2003). doi:10.1172/JCI200318921.

\section{Introduction}

In the mid 1990s, immunoassays for C-reactive protein (CRP), with greater sensitivity than those previously in routine use, revealed that increased CRP values, even within the range previously considered normal, strongly predict future coronary events. These findings triggered widespread interest, especially, remarkably, in the US, where the clinical use of CRP measurement had been largely ignored for about 30 years. CRP production is part of the nonspecific acute-phase response to most forms of inflammation, infection, and tissue damage and was therefore considered not to provide clinically useful information. Indeed, CRP values can never be diagnostic on their own and can only be interpreted at the bedside, in full knowledge of all other clinical and pathological results. However, they can then contribute powerfully to management, just as universal recording of the patient's temperature, an equally nonspecific parameter, is of great clinical utility.

The present torrent of studies of CRP in cardiovascular disease and associated conditions is facilitated by the ready commercial availability of automated CRP assays and of CRP itself as a research reagent. However, unlike the earlier rejection in the US of CRP as an empirical test because of its perceived lack of specificity, the current enthusiasm over CRP in cardiovascular disease is widely characterized by failure to recognize appropriately the nonspecific nature of the acutephase response, and by lack of critical biological judgment. Quality control of the source, purity, and structural and functional integrity of the CRP, and the relevance of experimental design before ascribing pathophysiological functions, are also often ignored.

This article provides information about CRP as a protein and an acute-phase reactant, and a knowledge-based framework for interpretation and analysis

\footnotetext{
Address correspondence to: Mark B. Pepys, Department of Medicine, Centre for Amyloidosis and Acute Phase Proteins, Royal Free and University College Medical School, Rowland Hill Street, London NW3 2PF, United Kingdom. Phone: 44-20-7433-2801; Fax: 44-20-7433-2803; E-mail: m.pepys@rfc.ucl.ac.uk.
}

Conflict of interest: The authors have declared that no conflict of interest exists.

Nonstandard abbreviations used: C-reactive protein (CRP); serum amyloid A protein (SAA); serum amyloid $\mathrm{P}$ component (SAP). of clinical observations of CRP in relation to cardiovascular and other diseases. We also review the properties of CRP, its possible role in pathogenesis of disease, and our own observations that identify it as a possible therapeutic target.

\section{The acute-phase response}

CRP, named for its capacity to precipitate the somatic C-polysaccharide of Streptococcus pneumoniae, was the first acute-phase protein to be described and is an exquisitely sensitive systemic marker of inflammation and tissue damage (1). The acute-phase response comprises the nonspecific physiological and biochemical responses of endothermic animals to most forms of tissue damage, infection, inflammation, and malignant neoplasia. In particular, the synthesis of a number of proteins is rapidly upregulated, principally in hepatocytes, under the control of cytokines originating at the site of pathology. Other acute-phase proteins include proteinase inhibitors and coagulation, complement, and transport proteins, but the only molecule that displays sensitivity, response speed, and dynamic range comparable to those of CRP is serum amyloid A protein (SAA) (Table 1) (1).

\section{Circulating CRP concentration}

In healthy young adult volunteer blood donors, the median concentration of CRP is $0.8 \mathrm{mg} / \mathrm{l}$, the 90 th centile is $3.0 \mathrm{mg} / 1$, and the 99th centile is $10 \mathrm{mg} / \mathrm{l}(2)$, but, following an acute-phase stimulus, values may increase from less than $50 \mu \mathrm{g} / 1$ to more than 500 $\mathrm{mg} / \mathrm{l}$, that is, 10,000-fold. Plasma CRP is produced only by hepatocytes, predominantly under transcriptional control by the cytokine IL-6, although other sites of local CRP synthesis and possibly secretion have been suggested. De novo hepatic synthesis starts very rapidly after a single stimulus, serum concentrations rising above $5 \mathrm{mg} / \mathrm{l}$ by about 6 hours and peaking around 48 hours. The plasma half-life of CRP is about 19 hours and is constant under all conditions of health and disease, so that the sole determinant of circulating CRP concentration is the synthesis rate (3), which thus directly reflects the intensity of the pathological process(es) stimulating CRP production. When the stimulus for increased production completely ceases, the circulating CRP concentration falls rapidly, at 
Table 1

Changes in concentrations of plasma proteins in the acute-phase response

\begin{tabular}{|c|c|c|}
\hline & Increased & Decreased \\
\hline Proteinase inhibitors & $\begin{array}{c}\alpha_{1} \text {-Antitrypsin } \\
\alpha_{1} \text {-Antichymotrypsin }\end{array}$ & Inter- $\alpha$-antitrypsir \\
\hline Coagulation proteins & $\begin{array}{l}\text { Fibrinogen } \\
\text { Prothrombin } \\
\text { Factor VIII } \\
\text { Plasminogen }\end{array}$ & \\
\hline Complement proteins & $\begin{array}{c}\text { C1s } \\
\text { C2 } \\
\text { B } \\
\text { C3 } \\
\text { C4 } \\
\text { C5 } \\
\text { C1 inhibitor }\end{array}$ & Properdin \\
\hline Transport proteins & $\begin{array}{l}\text { Haptoglobin } \\
\text { Hemopexin } \\
\text { Ceruloplasmin }\end{array}$ & \\
\hline Miscellaneous & $\begin{array}{c}\text { CRP } \\
\text { SAA } \\
\text { Fibronectin } \\
\alpha_{1} \text {-acid glycoprotein } \\
\text { Gc globulin }\end{array}$ & $\begin{array}{c}\text { Albumin } \\
\text { Transthyretin } \\
\text { HDL } \\
\text { LDL }\end{array}$ \\
\hline
\end{tabular}

almost the rate of plasma CRP clearance. In unselected general populations of ostensibly healthy subjects, the median CRP value is slightly higher than among blood donors and tends to increase with age, presumably reflecting the increasing incidence of subclinical pathologies (4). However, surprisingly in view of the sensitivity, speed, and range of the CRP response, subjects in the general population tend to have stable CRP concentrations characteristic for each individual, apart from occasional spikes presumably related to minor or subclinical infections, inflammation, or trauma. There is no significant seasonal variation in base-line CRP concentration, and, remarkably, the self correlation coefficient of measurements repeated years apart is about 0.5 , which is comparable to that of cholesterol. Twin studies show a highly significant hereditable component in base-line CRP values that is independent of age and BMI. Associations between CRP production and genetic polymorphisms in IL-1 and IL- 6 have been suggested, and a polymorphic GT repeat in the intron of the CRP gene is reportedly associated with differences in base-line CRP concentrations in normal individuals and in patients with systemic lupus erythematosus (5), and also with susceptibility to invasive pneumococcal disease. If such polymorphisms, particularly in the CRP gene itself, can be shown to reliably correlate with base-line CRP concentrations, and/or CRP production in the acute-phase response, and also with clinical outcome, the case for a pathogenetic and/or host-defense role of CRP in inflammatory disease will be strengthened.

In most, though not all, diseases (Table 2), the circulating value of CRP reflects ongoing inflammation and/or tissue damage much more accurately than do other laboratory parameters of the acute-phase response, such as plasma viscosity and the erythrocyte sedimentation rate. Importantly, acute-phase CRP values show no diurnal variation and are unaffected by eating. Liver failure impairs CRP production, but no other intercurrent pathologies and very few drugs reduce CRP values unless they also affect the underlying pathology providing the acute-phase stimulus. The CRP concentration is thus a very useful nonspecific biochemical marker of inflammation, measurement of which contributes importantly to (a) screening for organic disease, (b) monitoring of the response to treatment of inflammation and infection, and (c) detection of intercurrent infection in immunocompromised individuals, and in the few specific diseases characterized by modest or absent acute-phase responses (Table 3) (1). It is not known why systemic lupus erythematosus and the other conditions listed with it in Table 2 fail to elicit major CRP production despite evident inflammation and tissue damage, nor why the CRP responses to intercurrent infection are apparently intact in patients with such conditions.

\section{Structure and phylogeny of CRP}

CRP belongs to the pentraxin family of calciumdependent ligand-binding plasma proteins, the other member of which in humans is serum amyloid $\mathrm{P}$ component (SAP). The human CRP molecule $\left(\mathrm{M}_{\mathrm{r}} 115,135\right)$ is composed of five identical nonglycosylated polypeptide subunits ( $\mathrm{Mr} 23,027$ ), each containing 206 amino

\section{Table 2}

CRP responses in disease

Major CRP acute-phase response Infections

Allergic complications of infection

Inflammatory disease

Necrosis

Bacterial Systemic/Severe fungal, mycobacterial, viral Rheumatic fever Erythema nodosum

Rheumatoid arthritis Juvenile chronic arthritis

Ankylosing spondylitis Psoriatic arthritis Systemic vasculitis

Polymyalgia rheumatica Reiter disease Crohn disease Familial Mediterranean fever Myocardial infarction Tumor embolization Acute pancreatitis

Trauma Surgery Burns Fractures Malignancy Lymphoma Carcinoma Sarcoma

Modest or absent CRP acute-phase response

Systemic lupus erythematosus Scleroderma

Dermatomyositis

Ulcerative colitis Leukemia

Graft-versus-host disease 
Table 3

Routine clinical uses of CRP measurement

Screening test for organic disease

Assessment of disease activity in inflammatory conditions Juvenile chronic (rheumatoid) arthritis Rheumatoid arthritis

Ankylosing spondylitis

Reiter disease

Psoriatic arthropathy

Vasculitides Behçet syndrome

Wegener granulomatosis

Polyarteritis nodosa

Polymyalgia rheumatica

Crohn disease

Rheumatic fever

Familial fevers including familial Mediterranean fever

Acute pancreatitis

Diagnosis and management of infection

Bacterial endocarditis

Neonatal septicemia and meningitis

Intercurrent infection in systemic lupus erythematosus

Intercurrent infection in leukemia and its treatment

Postoperative complications including infection and thromboembolism

Differential diagnosis/classification of inflammatory disease

Systemic lupus erythematosus vs. rheumatoid arthritis Crohn disease vs. ulcerative colitis and even in the phylogenetically distant arachnid, Limulus polyphemus, the horseshoe crab. SAP, named for its universal presence in amyloid deposits, is a constitutive, non-acute-phase plasma glycoprotein in humans and all other species studied, except the mouse, in which it is the major acute-phase protein. In contrast, mouse CRP is a trace protein whose concentration increases only modestly, to a maximum of about $2 \mathrm{mg} / \mathrm{l}$, during the acute-phase response. No mouse CRP knockout, to our knowledge, has yet been made, and in vivo work on CRP function has largely been confined to passive administration of exogenous, heterologous CRP or to mice transgenic for rabbit or human CRP. These artifactual heterologous systems may not provide physiologically relevant information. Despite the evolutionary conservation of sequence, subunit organization, and protein fold, there are considerable variations between CRPs of different species with respect to fine ligand-binding specificity, presence and nature of glycosylation, protomer assembly, capacity to precipitate and aggregate ligands, base-line circulating concentrations, behavior as acute-phase proteins, and capacity to activate autologous complement (7-9). Indeed, only human CRP has been rigorously shown to activate complement in isologous serum. These differences command extreme caution in extrapolating from animal models to humans.

\section{Ligand binding and biological role of CRP}

Human CRP binds with highest affinity to phosphocholine residues, but it also binds to a variety of other autologous and extrinsic ligands, and it aggregates or precipitates the cellular, particulate, or molecular structures bearing these ligands. Autologous ligands include native and modified plasma lipoproteins (10), damaged cell membranes (11), a number of different phospholipids and related compounds, small nuclear ribonucleoprotein particles (12), and apoptotic cells (13). Extrinsic ligands include many glycan, phospholipid, and other constituents of microorganisms, such as capsular and somatic components of bacteria, fungi, and parasites, as well as $\mathbf{a}$

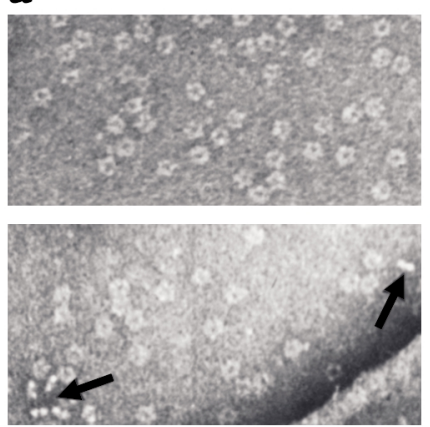

b

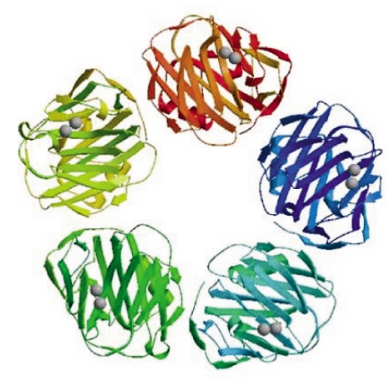

C

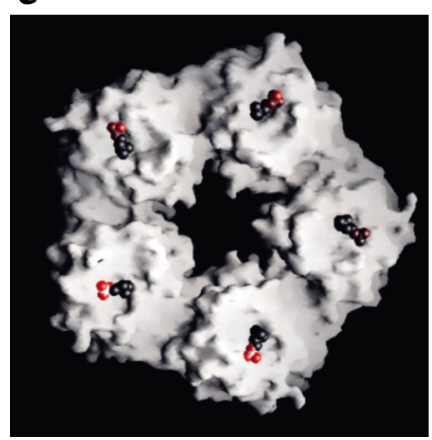

Figure 1

Molecular structure and morphology of human CRP. (a) Negatively stained electron micrograph showing the typical pentameric disc-like structure face-on and side-on (arrows). (b) Ribbon diagram of the crystal structure, showing the lectin fold and the two calcium atoms (spheres) in the ligand-binding site of each protomer (6). (c) Space-filling model of the CRP molecule, showing a single phosphocholine molecule located in the ligand-binding site of each protomer (6). 
plant products. When aggregated or bound to macromolecular ligands, human CRP is recognized by $\mathrm{C} 1 \mathrm{q}$ and potently activates the classical complement pathway, engaging $\mathrm{C} 3$, the main adhesion molecule of the complement system, and the terminal membrane attack complex, C5-C9 $(14,15)$. Bound CRP may also provide secondary binding sites for factor $\mathrm{H}$ and thereby regulate alternative-pathway amplification and C5 convertases.

The secondary effects of CRP that follow ligand binding resemble some of the key properties of antibodies, suggesting that under various circumstances CRP may contribute to host defense against infection, function as a proinflammatory mediator, and participate in physiological and pathophysiological handling of autologous constituents. Evidence of CRP functioning in these various roles is available from experimental animal models, but there is no rigorous information from physiological isologous systems. The absence of any known deficiency or protein polymorphism of human CRP, and the phylogenetic conservation of CRP structure and its ligand-binding specificity for phosphocholine and related substances, suggest that this protein must have had survival value. Microbial infection is a major driving force of change during evolution, and CRP has many features compatible with a role in innate immunity. In addition, the impaired CRP response in active systemic lupus (1) and the marked spontaneous antinuclear autoimmunity of SAP knockout mice (16) are compatible with the possibility that pentraxins function to prevent autoimmunity.

Phosphocholine is a component of many prokaryotes and is almost universally present in eukaryotes (17), and a substantial proportion of germline-encoded, highly conserved natural antibodies resemble CRP in specifically recognizing phosphocholine. The capacity to bind these residues may thus be important for both host defense and handling of autologous constituents (1) including necrotic (18) and apoptotic cells $(13,19)$. Activation of complement by human CRP may then opsonize and enhance phagocytosis of these various ligands but could also mediate proinflammatory pathophysiological effects (1). Intriguingly, the spectrum of autologous ligands recognized by CRP overlaps that of anti-phospholipid autoantibodies that are associated with premature cardiovascular disease in autoimmune syndromes.
Some functions that have been claimed for CRP seem inherently unlikely. For example, it is improbable that a plasma protein with a dynamic range of 10,000 -fold within hours would function like a cytokine or be a fine modulator of sophisticated cellular or physiological systems. Another implausible speculation concerns dissociated denatured CRP subunits, so-called modified or neo-CRP, for which various biological effects have been reported in vitro. Native CRP is actually very stable, and release of separate protomers requires exposure of the protein to harsh denaturing conditions. There is no compelling evidence for the persistence of denatured CRP in vivo, and rapid complete catabolism of such material would be expected.

\section{CRP and cardiovascular disease}

Earlier work suggested a prognostic association between increased CRP production and outcome after acute myocardial infarction (20) and in acute coronary syndromes (21). However, our original study with high sensitivity measurements of CRP in patients with severe unstable angina (22), and the European Concerted Action on Thrombosis and Disabilities Angina Pectoris Study of outpatients with both stable and unstable angina $(23,24)$, first drew attention to the predictive significance of such measurements for future coronary events. Analysis of CRP values in stored sera from large epidemiological studies rapidly followed, and the subsequent availability of routine high-sensitivity assays for CRP has enabled a flood of studies demonstrating a predictive relationship between increased CRP production and future atherothrombotic events, including coronary events, stroke, and progression of peripheral arterial disease (25-30). Meta-analysis of all published studies up to the year 2000, comprising a total of 1,953 coronary events, showed a relative risk of 2.0 for a future coronary event in subjects with a single initial base-line CRP value in the upper third compared with those in the lower third of the distribution in the general population (29) (see "CRP and atherothrombotic events"). There have been further studies comprising 2,648 coronary events up to the year 2002, and the meta-analysis of these, together with the Reykjavik Icelandic Heart Study of about 19,000 individuals including 2,459 coronary-event patients

\section{CRP and atherothrombotic events}

Raised base-line CRP values predict coronary events, stroke, and progression of peripheral disease

Relative risk of coronary event is 2.0 for single base-line CRP concentration $>2.4 \mathrm{mg} / \mathrm{l}$ versus $<1 \mathrm{mg} / \mathrm{l}$

What is driving this increased CRP production?

- Inflammation in atherosclerotic plaques?

- Total atherosclerotic burden?

- Inflammation elsewhere in the body?

- Metabolic status rather than inflammation?

- Individual higher CRP responsiveness to prevalent endogenous or environmental acute-phase stimuli?

CRP is not uniquely associated with cardiovascular disease; other systemic markers of inflammation show similar associations, though less marked ones than that of CRP 
who underwent follow-up for almost 20 years, is currently in preparation and will provide the most robust estimate to date of the relative risk predicted by CRP. The recent emphasis in cardiovascular medicine on "high-sensitivity" or "highly sensitive" CRP, abbreviated as so-called hs-CRP, seems to have created a false impression in some quarters that this is somehow a different analyte from "conventional" CRP. This is incorrect. The "high sensitivity" refers simply to the lower detection limit of the assay procedures being used. The actual CRP analyte, the plasma protein that is being measured, is the same regardless of the assay range. Very sensitive CRP assays have been reported from research laboratories for 30 years (1). The new development is the introduction of commercial and automated routine CRP immunoassay systems with greater sensitivity than before.

Circulating CRP values correlate closely with other markers of inflammation, some of which show similar, albeit generally less significant, predictive associations with coronary events $(31,32)$. The attention focused on CRP reflects in part the fact that it is an exceptionally stable analyte in serum or plasma and that immunoassays for it are robust, well standardized, reproducible, and readily available. Furthermore, the intrinsic biological properties of CRP as an acute-phase reactant are, as explained above, especially favorable for its use as a sensitive quantitative systemic readout of the acute-phase response. In contrast, none of the other systemic markers of inflammation, whether upstream cytokine mediators, other sensitive acute-phase proteins such as SAA, negative acute-phase proteins such as albumin, or cruder multifactorial measures such as erythrocyte sedimentation rate or polymorph count, has such robust and desirable characteristics. The inherent properties of CRP and its behavior may sufficiently explain why it provides closer associations and better predictions than other markers of inflammation. However, CRP may also have specific associations with cardiovascular disease, as discussed below.

\section{Atherosclerosis and inflammation}

The mechanisms responsible for the low-grade upregulation of CRP production that predicts coronary events in general populations (27-29) are unknown (see "CRP and atherothrombotic events"). The causes of the often more substantial CRP values associated with poor prognosis in severe unstable angina (22) or after angioplasty are also obscure. Atherosclerosis, and the evolution of plaque instability underlying atherothrombotic events, are inflammatory processes. It has thus been widely assumed, without direct supporting evidence, that the relevant acute-phase stimuli arise from inflammation within atheromatous lesions themselves and reflect their extent and/or severity. This is certainly possible, although there are conflicting observations regarding the association between CRP values and various indirect measures of atheroma burden. However, chronic systemic, nonvascular inflammation is known to be proatherogenic in general, and acute systemic inflammatory episodes are strongly associated with atherothrombotic events. The increased production of CRP that predicts atherothrombotic events may therefore reflect inflammation elsewhere in the body, although there is no correlation with serological evidence of the various chronic microbial infections, such as Chlamydia pneumoniae and Helicobacter pylori, that have been putatively linked with coronary heart disease (29). Another possibility is that individuals vary in their sensitivity to the general background of intercurrent low-grade acute-phase stimuli to which everybody is exposed, and that those who are higher "CRP responders," through genetic and/or acquired mechanisms, are also more susceptible to progression and complications of atherosclerosis, regardless of whether there is a causal relationship.

There is a strong positive association between baseline CRP concentration and BMI (32), and weight loss lowers the CRP value. Raised base-line CRP values are also associated with many features of the insulin resistance or metabolic syndrome $(33,34)$, up to and including frank diabetes mellitus (35). This may reflect, in part, the fact that adipocytes are the source of a substantial portion of base-line IL-6 production (36) and perhaps also synthesize and secrete some of the baseline CRP itself. More generally, these associations raise the possibility that aspects of the inflammatory-marker profile associated with increased atherothrombotic risk in the population at large may not be triggered by inflammation or tissue damage in the classical sense. Rather, they may reflect a particular metabolic state that happens also to be proatherogenic and/or to predispose to atherothrombotic events. Indeed, CRP production predicts the development of type 2 diabetes independently of traditional risk factors (37). In insulin-resistant obese individuals, elevated CRP values fall in parallel with improvements in insulin resistance 
that are associated with weight loss, but the association between CRP and insulin resistance is independent of body mass (38). Other potentially important physiological rather than pathophysiological influences are suggested by the finding that oral contraceptive use (39) and systemic, but not transdermal, postmenopausal hormone replacement therapy $(40,41)$ are also associated with significantly raised base-line CRP concentrations without any sign of tissue-damaging inflammation. Similarly, physical exercise (42) and moderate alcohol consumption (43) are both associated with lowering of base-line CRP concentration. In contrast, the positive association of CRP values with other classical cardiovascular disease risk factors, such as periodontal disease and smoking (32), seems more clearly related to local nonarterial inflammation.

HMG CoA-reductase inhibitors, the drugs known as statins, reproducibly reduce CRP values, independently of their effects on lipid profiles (44). It is not known whether this reflects direct effects on hepatocytes, anti-inflammatory effects within atherosclerotic plaques, and/or anti-inflammatory effects elsewhere. However, recent studies suggest that statins reduce the risk of future cardiovascular events to the same extent in patients with raised LDL cholesterol values and in those with normal LDL but with baseline CRP concentrations above the median (45). If these observations are confirmed, measurement of CRP may become an indication for prophylactic antiatherosclerotic therapy in otherwise apparently low-risk individuals and populations.

However, it is critically important to recognize that the CRP response is nonspecific and is triggered by many disorders unrelated to cardiovascular disease (Table 2). In using CRP for assessment of cardiovascular risk, it is therefore essential to clearly establish true base-line CRP values that are not distorted by either trivial or serious intercurrent pathologies. If the initial CRP result is in the low-risk range, less than $1 \mathrm{mg} / \mathrm{l}$, a single measurement is sufficient, but if it is in the higher-risk range, less than about $2.5 \mathrm{mg} / \mathrm{l}$, two or more serial samples taken at intervals of 1 week or more should be retested until a stable base-line value is seen. If the CRP value persistently remains above $10 \mathrm{mg} / \mathrm{l}$, indicating the presence of a significant acute-phase response, a full history and physical examination of the patient is indicated, ideally together with relevant investigations, to determine the cause and alleviate it if possible. Interestingly, chronic inflammatory conditions, such as rheumatoid arthritis and hemodialysis for end-stage renal failure, that are characterized by persistently elevated CRP concentrations in some individuals, are associated with premature cardiovascular disease.

\section{CRP and pathogenesis of atherosclerosis?}

Binding of CRP to lipids, especially lecithin (phosphatidyl choline), and to plasma lipoproteins has been known for over 60 years, but the first suggestion of a possible relationship to atherosclerosis came when we demonstrated that aggregated, but not native, nonaggregated, CRP selectively bound only LDL and some VLDL from whole serum $(10,46)$. However, native CRP does bind to oxidized LDL (19) and to partly degraded LDL, as found in atheromatous plaques, and then activates complement (47). Furthermore, CRP is present in most such plaques examined ex vivo $(48,49)$. This CRP could promote complement activation and thus inflammation in the plaques, and there is experimental evidence supporting a possible role of complement in atherogenesis (see "Possible specific associations of CRP with cardiovascular disease"). Addition of CRP to LDL in cell culture systems has been reported to stimulate formation of foam cells, which are a typical feature of atherosclerotic plaques (50). It is not known whether this reflects opsonization of the LDL particles by CRP or an effect of CRP on the phagocytic cells themselves. It has been claimed that CRP is recognized by a subset of cellular Fc $\gamma$ receptors and could thereby directly opsonize its ligands and/or engage multiple processes of inflammation. However, robustly controlled studies, using recombinant and highly purified human CRP and avoiding use of whole IgG anti-CRP antibodies, do not confirm such interactions with human cells $(51,52)$.

CRP has also been reported to stimulate tissue factor production by peripheral blood monocytes in vitro and could thereby have important procoagulant effects (53). Other studies show a variety of effects in cell cultures exposed to CRP, including, for example, increased expression of adhesion molecules, and modulation of NO synthesis. However, these experiments have generally used commercially sourced CRP of incompletely defined provenance and purity, and there have been few robust specificity controls. The findings must be treated with caution until the purity and structural and functional integrity of the CRP have been rigorously established and the specificity of the observed effects confirmed, for example by comparison with appropriate control proteins and by the use of specific CRP absorbents, ligands, antibodies, and inhibitors of binding.

\section{CRP and myocardial infarction}

Tissue necrosis is a potent acute-phase stimulus, and, following myocardial infarction, there is a major CRP response, the magnitude of which reflects the extent of myocardial necrosis (20). Furthermore, the peak CRP values at around 48 hours after the onset powerfully predict outcome after myocardial infarction (20,54-56). Importantly, CRP is co-deposited with activated complement within all acute myocardial infarcts $(57,58)$, and compelling experimental evidence now suggests that the CRP response not only reflects tissue damage in this context but may also contribute significantly to the severity of ischemic myocardial injury (59).

We specifically investigated in vivo the complementdependent proinflammatory role of human CRP in rats, taking advantage of the fact that rat CRP does 
not activate rat complement while human CRP potently activates both rat and human complement (8). When rats that had undergone coronary artery ligation received daily injections of pure human CRP, they became sicker than similarly operated rats that received buffer alone or the closely related pentraxin human SAP, which does not activate complement (59). Injection of human CRP into unoperated rats had no adverse effects. Some of the coronary artery-ligated rats treated with human CRP died, and those that survived to day 5 , when all animals were killed, had infarcts $40 \%$ larger than those of buffer- or SAP-treated controls (59). This dramatic enhancement of infarct size by human CRP was completely abrogated by in vivo complement depletion of the rats using cobra venom factor, and hence it was absolutely complement dependent (59).

\section{CRP: a target for therapy in human disease?}

We have long speculated that CRP may have significant proinflammatory effects, and that, by binding to ligands exposed on cells or other autologous structures as a result of infection, inflammation, ischemia, and other pathologies, and triggering complement activation, it may exacerbate tissue damage, leading to more severe disease (1). The rat myocardial infarction model provided the first direct evidence of these processes in vivo (59), but they are not necessarily confined to cardiovascular disease. The excellent correlation of circulating CRP concentrations with the severity, extent, and progression of many different pathologies, and the prognostic significance of these associations, are consistent with CRP not just being a marker of disease but also contributing to pathogenesis. A definitive way to test this concept will be the use of novel drugs that specifically block CRP binding and its proinflammatory effects in vivo (60). If these compounds are effective, they may find very broad applicability. Such drugs would be a powerful tool for determining whether increased CRP production merely reflects atherosclerosis or does indeed participate in its pathogenesis and complications, and they could also have cardioprotective effects in acute myocardial infarction. Knowledge of the structure and function of CRP - including its threedimensional structure alone and complexed with ligands (6) - coupled with experience in developing an inhibitor of the related protein SAP (61) establishes an excellent platform for drug design.

\section{Acknowledgments}

G.M. Hirschfield is a Medical Research Council Clinical Training Fellow. The work of the Centre for Amyloidosis and Acute Phase Proteins is supported by grants from the Medical Research Council (United Kingdom), the Wellcome Trust, and the Wolfson Foundation, and by National Health Service Research and Development Funds. We thank Beth Jones for expert assistance in preparing the manuscript.
1. Pepys, M.B., and Baltz, M.L. 1983. Acute phase proteins with special reference to C-reactive protein and related proteins (pentaxins) and serum amyloid A protein. Adv. Immunol. 34:141-212.

2. Shine, B., de Beer, F.C., and Pepys, M.B. 1981. Solid phase radioimmunoassays for C-reactive protein. Clin. Chim. Acta. 117:13-23.

3. Vigushin, D.M., Pepys, M.B., and Hawkins, P.N. 1993. Metabolic and scintigraphic studies of radioiodinated human C-reactive protein in health and disease. J. Clin. Invest. 91:1351-1357.

4. Hutchinson, W.L., et al. 2000. Immunoradiometric assay of circulating C-reactive protein: age-related values in the adult general population. Clin. Chem. 46:934-938.

5. Szalai, A.J., McCrory, M.A., Cooper, G.S., Wu, J., and Kimberly, R.P. 2002. Association between baseline levels of C-reactive protein (CRP) and a dinucleotide repeat polymorphism in the intron of the CRP gene. Genes Immun. 3:14-19.

6. Thompson, D., Pepys, M.B., and Wood, S.P. 1999. The physiological structure of human C-reactive protein and its complex with phosphocholine. Structure. 7:169-177.

7. Oliveira, E.B., Gotschlich, E.C., and Liu, T.-Y. 1980. Comparative studies on the binding properties of human and rabbit C-reactive proteins. J. Immunol. 124:1396-1402.

8. de Beer, F.C., et al. 1982. Isolation and characterisation of C-reactive protein and serum amyloid $\mathrm{P}$ component in the rat. Immunology. 45:55-70.

9. Baltz, M.L., et al. 1982. Phylogenetic aspects of C-reactive protein and related proteins. Ann. N. Y. Acad. Sci. 389:49-75.

10. Pepys, M.B., Rowe, I.F., and Baltz, M.L. 1985. C-reactive protein: binding to lipids and lipoproteins. Int. Rev. Exp. Pathol. 27:83-111.

11. Volanakis, J.E., and Wirtz, K.W.A. 1979. Interaction of C-reactive protein with artificial phosphatidylcholine bilayers. Nature. 281:155-157.

12. Du Clos, T.W. 1989. C-reactive protein reacts with the U1 small nuclear ribonucleoprotein. J. Immunol. 143:2553-2559.

13. Gershov, D., Kim, S., Brot, N., and Elkon, K.B. 2000. C-reactive protein binds to apoptotic cells, protects the cells from assembly of the terminal complement components, and sustains an antiinflammatory innate immune response: implications for systemic autoimmunity. J. Exp. Med. 192:1353-1363.

14. Volanakis, J.E. 1982. Complement activation by C-reactive protein complexes. Ann. N. Y. Acad. Sci. 389:235-250.

15. Mold, C., Gewurz, H., and Du Clos, T.W. 1999. Regulation of complement activation by C-reactive protein. Immunopharmacology. 42:23-30.

16. Bickerstaff, M.C.M., et al. 1999. Serum amyloid P component controls chromatin degradation and prevents antinuclear autoimmunity. Nat. Med. 5:694-697.

17. Harnett, W., and Harnett, M.M. 1999. Phosphorylcholine: friend or foe of the immune system? Immunol. Today. 20:125-129.

18. Kushner, I., and Kaplan, M.H. 1961. Studies of acute phase protein. I. An immunohistochemical method for the localization of Cx-reactive protein in rabbits. Association with necrosis in local inflammatory lesions. J. Exp. Med. 114:961-973.

19. Chang, M.K., Binder, C.J., Torzewski, M., and Witztum, J.L. 2002. C-reactive protein binds to both oxidized LDL and apoptotic cells through recognition of a common ligand: phosphorylcholine of oxidized phospholipids. Proc. Natl. Acad. Sci. U. S. A. 99:13043-13048.

20. de Beer, F.C., et al. 1982. Measurement of serum C-reactive protein concentration in myocardial ischaemia and infarction. Br. Heart $J$. 47:239-243.

21. Berk, B.C., Weintraub, W.S., and Alexander, R.W. 1990. Elevation of C-reactive protein in "active" coronary artery disease. Am. J. Cardiol. 65:168-172.

22. Liuzzo, G., et al. 1994. The prognostic value of C-reactive protein and serum amyloid A protein in severe unstable angina. N. Engl. J. Med. 331:417-424.

23. Thompson, S.G., Kienast, J., Pyke, S.D.M., Haverkate, F., and van de Loo, J.C.W. 1995. Hemostatic factors and the risk of myocardial infarction or sudden death in patients with angina pectoris. N. Engl. J. Med. 332:635-641.

24. Haverkate, F., Thompson, S.G., Pyke, S.D.M., Gallimore, J.R., and Pepys, M.B. 1997. Production of C-reactive protein and risk of coronary events in stable and unstable angina. Lancet. 349:462-466.

25. Kuller, L.H., Tracy, R.P., Shaten, J., and Meilahn, E.N. 1996. Relation of $\mathrm{C}$-reactive protein and coronary heart-disease in the MRFIT nested case control study. Am. J. Epidemiol. 144:537-547.

26. Tracy, R.P., et al. 1996. C-reactive protein and incidence of cardiovascular disease in older women: the rural health promotion project and the cardiovascular health study. Circulation. 93:622.

27. Ridker, P.M., Cushman, M., Stampfer, M.J., Tracy, R.P., and Hennekens, C.H. 1997. Inflammation, aspirin, and the risk of cardiovascular disease in apparently healthy men. N. Engl. J. Med. 336:973-979.

28. Koenig, W., et al. 1999. C-reactive protein, a sensitive marker of inflammation, predicts future risk of coronary heart disease in initially healthy middle-aged men: results from the MONICA (Monitoring Trends and 
Determinants in Cardiovascular Disease) Augsburg Cohort Study, 1984 to 1992. Circulation. 99:237-242.

29. Danesh, J., et al. 2000. Low grade inflammation and coronary heart disease: prospective study and updated meta-analyses. BMJ. 321:199-204.

30. Ridker, P.M., Hennekens, C.H., Buring, J.E., and Rifai, N. 2000. C-reactive protein and other markers of inflammation in the prediction of cardiovascular disease in women. N. Engl. J. Med. 342:836-843.

31. Danesh, J., Collins, R., Appleby, P., and Peto, R. 1998. Association of fibrinogen, C-reactive protein, albumin, or leukocyte count with coronary heart disease. J. Am. Coll. Cardiol. 279:1477-1482.

32. Danesh, J., et al. 1999. Risk factors for coronary heart disease and acutephase proteins. A population-based study. Eur. Heart J. 20:954-959.

33. Fröhlich, M., et al. 2000. Association between C-reactive protein and features of the metabolic syndrome: a population-based study. Diabetes Care. 23:1835-1839.

34. Chambers, J.C., et al. 2001. C-reactive protein, insulin resistance, central obesity, and coronary heart disease risk in Indian Asians from the United Kingdom compared with European whites. Circulation. 104:145-150.

35. Ford, E.S. 1999. Body mass index, diabetes, and C-reactive protein among U.S. adults. Diabetes Care. 22:1971-1977.

36. Yudkin, J.S., Stehouwer, C.D.A., Emeis, J.J., and Coppack, S.W. 1999. C-reactive protein in healthy subjects: associations with obesity, insulin resistance, and endothelial dysfunction. A potential role for cytokines originating from adipose tissue? Arterioscler. Thromb. Vasc. Biol. 19:972-978

37. Freeman, D.J., et al. 2002. C-reactive protein is an independent predictor of risk for the development of diabetes in the West of Scotland Coronary Prevention Study. Diabetes. 51:1596-1600.

38. McLaughlin, T., et al. 2002. Differentiation between obesity and insulin resistance in the association with C-reactive protein. Circulation. 106:2908-2912.

39. Fröhlich, M., et al. 1999. Oral contraceptive use is associated with a systemic acute phase response. Fibrinolysis and Proteolysis. 13:239-244.

40. Cushman, M., et al. 1999. Effect of postmenopausal hormones on inflammation-sensitive proteins: the Postmenopausal Estrogen/Progestin Interventions (PEPI) Study. Circulation. 100:717-722.

41. Ridker, P.M., Hennekens, C.H., Rifai, N., Buring, J.E., and Manson, J.E. 1999. Hormone replacement therapy and increased plasma concentration of C-reactive protein. Circulation. 100:713-716.

42. Ford, E.S. 2002. Does exercise reduce inflammation? Physical activity and C-reactive protein among U.S. adults. Epidemiology. 13:561-568.

43. Imhof, A., et al. 2001. Effect of alcohol consumption on systemic markers of inflammation. Lancet. 357:763-767.

44. Ridker, P.M., Rifai, N., Pfeffer, M.A., Sacks, F., and Braunwald, E. 1999. Long-term effects of pravastatin on plasma concentration of C-reactive protein. Circulation. 100:230-235.

45. Ridker, P.M., et al. 2001. Measurement of C-reactive protein for the targeting of statin therapy in the primary prevention of acute coronary events. N. Engl. J. Med. 344:1959-1965.
46. de Beer, F.C., et al. 1982. Low density and very low density lipoproteins are selectively bound by aggregated C-reactive protein. J. Exp. Med. 156:230-242.

47. Bhakdi, S., Torzewski, M., Klouche, M., and Hemmes, M. 1999. Com plement and atherogenesis. Binding of CRP to degraded, nonoxidized LDL enhances complement activation. Arterioscler. Thromb. Vasc. Biol. 19:2348-2354

48. Torzewski, J., et al. 1998. C-reactive protein frequently colocalizes with the terminal complement complex in the intima of early atherosclerotic lesions of human coronary arteries. Arterioscler. Thromb. Vasc. Biol. 18:1386-1392.

49. Zhang, Y.X., Cliff, W.J., Schoefl, G.I., and Higgins, G. 1999. Coronary C-reactive protein distribution: its relation to development of atherosclerosis. Atherosclerosis. 145:375-379.

50. Zwaka, T.P., Hombach, V., and Torzewski, J. 2001. C-reactive proteinmediated low density lipoprotein uptake by macrophages: implications for atherosclerosis. Circulation. 103:1194-1197.

51. Hundt, M., Zielinska-Skowronek, M., and Schmidt, R.E. 2001. Lack of specific receptors for C-reactive protein on white blood cells. Eur. J. Immunol. 31:3475-3483.

52. Saeland, E., et al. 2001. Human C-reactive protein does not bind to FcgammaRIIa on phagocytic cells. J. Clin. Invest. 107:641-643.

53. Cermak, J., et al. 1993. C-reactive protein induces human peripheral blood monocytes to synthesize tissue factor. Blood. 82:513-520.

54. Pietilä, K.O., Harmoinen, A.P., Jokiniitty, J., and Pasternack, A.I. 1996. Serum C-reactive protein concentration in acute myocardial infarction and its relationship to mortality during 24 months of follow-up in patients under thrombolytic treatment. Eur. Heart J. 17:1345-1349.

55. Ueda, S., et al. 1996. C-reactive protein as a predictor of cardiac rupture after acute myocardial infarction. Am. Heart J. 131:857-860.

56. Anzai, T., et al. 1997. C-reactive protein as a predictor of infarct expansion and cardiac rupture after a first Q-wave acute myocardial infarction. Circulation. 96:778-784.

57. Kushner, I., Rakita, L., and Kaplan, M.H. 1963. Studies of acute phase protein. II. Localization of Cx-reactive protein in heart in induced myocardial infarction in rabbits. J. Clin. Invest. 42:286-292.

58. Lagrand, W.K., et al. 1997. C-reactive protein colocalizes with complement in human hearts during acute myocardial infarction. Circulation. 95:97-103.

59. Griselli, M., et al. 1999. C-reactive protein and complement are important mediators of tissue damage in acute myocardial infarction. J. Exp. Med. 190:1733-1739.

60. Pepys, M.B. 1999. The Lumleian Lecture. C-reactive protein and amyloidosis: from proteins to drugs? In Horizons in medicine. Volume 10. G. Williams, editor. Royal College of Physicians of London. London, United Kingdom. 397-414.

61. Pepys, M.B., et al. 2002. Targeted pharmacological depletion of serum amyloid P component for treatment of human amyloidosis. Nature. 417:254-259. 